\title{
LENGUAJE, DEPRESIÓN Y CAMBIO
}

\author{
Isabel Caro Gabalda \\ Universidad de Valencia
}

\begin{abstract}
RESUMEN
En este trabajo seguimos la perspectiva lingüística de la semántica general, aplicada a la conceptualización y análisis de parte de la experiencia depresiva a través de la terapia linguística de evaluación. Articulamos todo el trabajo alrededor de la conceptualización y cambio en el proceso evaluador de una paciente diagnosticada con "depresión mayor". Se hace un especial hincapié en las consecuencias para nuestra sanidad de seguir una orientación intensional y en las ventajas de desarrollar otra de tipo extensional. Para exponer el cambio en las evaluaciones se utilizó la metodología del análisis de tareas, muy empleada dentro del paradigma de investigación de procesos para analizar de forma intensiva acontecimientos significativos de cambio
\end{abstract}

Palabras clave: terapia lingüistica de evaluación, evaluación, orientación intensional, orientación extensional, análisis de tareas

\begin{abstract}
The main aim of this paper is to conceptualize from a general semantics perspective, more concretely the linguistic therapy of evaluation, part of the depressive experience. We frame the whole paper around the conceptualization and change in the evaluating process of a patient diagnosed as having "major depression". We emphasize the consequences for our sanity of following an intensional orientation and the advantages of developing an extensional one. The task analysis methodology was used to explain the change obtained. This methodology is used within a process research paradigm to intensively analyzed significant change events.
\end{abstract}

Key words: linguistic therapy of evaluation, evaluation, intensional orientation, extensional orientation, task analysis. 


\section{INTRODUCCIÓN}

La finalidad de este trabajo no es la de plantear la relevancia que tiene el lenguaje para la psicoterapia, puesto que todos podemos asumir algo obvio: la mayor parte de 10 que hacemos, es realizado a través del lenguaje. En su lugar, pretendemos plantear una perspectiva lingüistica para conceptualizar la depresión, mostrando, sobre todo, cómo producir cambios língüísticos en una paciente "depresiva".

Dos son los marcos fundamentales que articulan este trabajo. En primer lugar, el marco conceptual: la terapia lingüistica de evaluación, surgida de la teoría de la semántica general, y en segundo lugar, el marco metodológico: el análisis de tareas, utilizado en psicoterapia dentro del paradigma de la investigación de procesos.

\section{EL LENGUAJE SEGÚN LA TEORÍA DE LA SEMÁNTICA GENERAL}

Desde la teoría de la semántica general desarrollada por Korzybski (1933) asumimos que los seres humanos somos una clase de vida simbólica, cuya más relevante característica es la de enlazar símbolos y transmitirlos de generación en generación (Korzybski, 1924). Quizá el aforismo de Korzybski (1924):"quién controla los simbolos nos controla" refleje, claramente, la meta de la teoría de la semántica general.

Tal y como lo planteó Korzybski (1933) un simbolo es un signo que representa algo. Aunque estén relacionados, en comparación con los símbolos, los signos son más estáticos, fijos, tienen una conexión necesaria con un referente, señalizan e indican algo, mientras que los simbolos son convencionales, y muy variables, sus conexiones con los referentes son totalmente arbitrarias, transmiten mensajes, y nos comunican cosas que no "están presentes". Por ejemplo, los cambios de luz de un semáforo serían signos, que por convención social simbolizan y prescriben normas importantes de comportamiento, pararnos, cruzar, arrancar, etc.

Por este motivo, la semántica general busca convertir al ser humano en crítico de los simbolos que usa para hablar de si mismo y estructurar todas sus experiencias.
Indudablemente la simbolización es una capacidad humana única y básica para nuestra supervivencia, puesto que mediante los símbolos guiamos nuestras acciones y hemos asentado nuestra civilización. Los símbolos son, pues, nuestras "armas" de pensamiento y comunicación. Ahora bien, ante los símbolos la semántica general aconseja que tengamos una serie de precauciones. En primer lugar, que mantengamos una independencia entre los que se simboliza y el símbolo. En segundo lugar, que reconozcamos que podemos crear símbolos para una gran variedad de "cosas": procesos, objetos, acontecimiento, reacciones ante lo que observamos, etc. $Y$ finalmente, que los símbolos dependen de nuestro ambiente cultural y semántico.

\section{LA DEPRESIÓN DESDE LA SEMÁNTICA GENERAL}

Cómo entendemos la depresión desde esta perspectiva. Veamos algunos ejemplos que sustentan la hipótesis de la independencia entre lo simbolizado y el símbolo.

Podemos estar de acuerdo en que la melancolia, la tristeza, la preocupación, caracterizan a la depresión, pero ésta ha sido simbolizada de forma diferente en diversas épocas (ejemplos en Arieti y Bemporad, 1979; Pérez, 1990).

Así para Aristóteles (384-322) (siguiendo a Hipócrates), la melancolía era consecuencia de una mezcla inusual de bilis negra. Para Isidoro de Sevilla (560-636), la desesperanza se derivaba del pecado. La receta obvia, la confesión. Las doctrinas psicosomáticas postuladas por Francisco Valles (Sagrada filosofia, 1587), defienden siguiendo el capitulo 35 de los Proverbios): "Dad sidra a los tristes y vino a los que sufran amargura, beban y olviden su necesidad y no recuerden más su dolor".

Según Miguel Sabuco (Nueva filosofia de la naturaleza del hombre, 1587),

"La congoja y preocupación se ha de tratar de acuerdo a una agenda, dejando los cuidados "a tiempos, y ponerlos en su lugar, como en un papel, haciendo lista, y fijarla en la pared, y alivia la congoja, y miedo de la memoria, y sin pena se miran alli los cuidados, y se hacen, y a la noche se duerme mejor. La gran 
congoja se aliviará con razones del alma, lo que es, ya es, o lo que ha de ser, será, mi fatiga no la mejora, ni remedia".

La melancolía en la época romántica se consideraba consecuencia de no ser comprendido ni apreciado por otros seres humanos. El sufrimiento y la decepción conducian al héroe romántico al suicidio. Recordemos la ola de suicidios en Europa por la publicación del libro de Goethe (1749-1832), "Las cuitas del joven Werther" (1774).

¿Cómo se considera hoy en día la depresión? Desde los trabajos iniciales de Freud, pasando por el humanismo, el conductismo, las terapias cognitivas hasta la cultura del Prozac, han pasado muchos años, miles de autores, múltiples simbolizaciones de la experiencia depresiva. Veamos una de estas perspectivas: la terapia lingüistica de evaluación.

\section{La depresión según la terapia lingüistica de evaluación}

La terapia lingüistica de evaluación (TLE) se podria definir como una forma de tratamiento que sigue los principios de la semántica general y cuya finalidad principal es lograr que los pacientes diferencien entre lo que experimentan y las palabras que aportan a aquello que experimentan. Fundamentalmente, es un método educativo y reeducativo que pone en práctica las derivaciones clínicas de las tres premisas no-aristotélicas, centro de la teoría de la semántica general, y que son (Korzybski, 1933): 1) el mapa no es el territorio o la palabra no es el objeto; 2) el mapa no cubre todo el territorio, o el conocimiento humano es incompleto; 3 ) siempre podemos hacer un mapa que incluya otro mapa, etc, o el lenguaje es autorreflexivo.

Mediante la primera premisa entendemos que los problemas emocionales son una consecuencia de no seguir el orden natural de evaluación, es decir, primero hechos y después palabras y de identificar palabras con hechos, en consecuencia trabajamos con los pacientes para que logren no identificar $y$ controlar sus anticipaciones y predicciones (ver orientación intensional y extensional más adelante). Según la segunda premisa se toman los problemas emocionales como problemas de conocimiento entendiéndose el cono- cimiento como abstracción. Trabajamos con los pacientes enseñándoles a reconocer y seguir el proceso de abstracción, evitando saltar a conclusiones y les enseñamos a lograr conclusiones más ajustadas estructuralmente a hechos (ver descripción de la técnica de los órdenes de abstracción más adelante). Finalmente, la tercera premisa nos permite entender la multiordinalidad de nuestras reacciones que son distintas según el nivel no verbal y verbal en el que nos movamos. Una derivación importante de ello es la conceptualización de los problemas de segundo orden -ansiedad por la ansiedad, miedo al miedo (Lee, 1952), como siendo causados por la multiordinalidad del lenguaje.

La TLE se puede incluir dentro de las actuales terapias cognitivas aunque tiene una serie de diferencias con ellas y una conceptualización propia de los "trastornos emocionales" consecuencia de su marco semánticogeneral (Caro, 1990a, 1994, 1996a).

Qué nos han contado sobre la depresión los terapeutas cognitivos. Que los pacientes piensan mal (aunque hay voces que demuestran que no tan mal, o en todo caso que los normales no son tan normales), que cometen una serie de errores cognitivos, como por ejemplo, sentirse los culpables de todo, con pensamientos absolutistas del tipo, "no hago nada bien", etc. $Y$ visiones negativas del presente, el si mismo y el futuro.

Para un terapeuta lingüistico, en la óptica de la Semántica general los así llamados depresivos, no piensan ni mejor, ni peor que otro tipo de personas, sino que se asume que las personas utilizamos el lenguaje de una forma especial y los pacientes, depresivos o de otro tipo, serían aquellos cuyas evaluaciones les son más dolorosas, cuando valoran situaciones de forma negativa, las rechazan, etc. Todos los seres humanos evalúamos, lo hacemos sobre nuestras experiencias, lo que sentimos, $y$ les damos significado. Cuando to que sentimos y lo que sobre ello decimos son dispares, tenemos problemas.

Me gustaría señalar que mis comentarios siguientes no suponen, en modo alguno, una nueva propuesta o una nueva lectura de la psicopatología. Nos movemos a otro nivel, ofreciendo un enfoque para estudiar cambios en pacientes deprimidos. 
En este sentido, lo que vamos a realizar es un microanálisis de un acontecimiento significativo de una paciente depresiva. Empezamos entendiendo la depresión como una cuestión de evaluación y en este microanálisis, asumimos que los pacientes a los que se les adscribe la etiqueta de depresión ( $y$ de otro tipo también) realizan dos tipos básicos de evaluaciones: no verbales y verbales.

\section{El proceso de evaluación}

La evaluación es un proceso muy complejo que puede abarcar niveles verbales y noverbales, implicando por igual al 'pensamiento' $y$ al 'sentimiento'. Sin embargo, las evaluaciones que hace una persona se manifiestan lingüísticamente y con éstas son con las que trabajamos directamente, ya que el uso del lenguaje es el medio del cambio.

Tendriamos, pues, tres niveles, que en cierta medida se pueden reducir a dos, lo que sentimos y su manifestación en palabras:

1) el proceso evaluativo, en sí, de un paciente, o la "primera" evaluación, por ejemplo, cuando un paciente depresivo "siente tristeza"

2) cómo lo relata, lo narra o lo manifiesta por escrito ya sea a él mismo o al terapeuta, y

3) posteriores evaluaciones a todo ello, que en cierta medida serían otros procesos evaluativos. Por ejemplo, cuando un paciente se pregunta ¿por qué estoy triste?, reconoce que no puede soportar el sentirme así, etc.

Clínicamente trabajamos con las evaluaciones verbales para afectar las no verbales. Entendiendo siempre que el organismo funciona como un todo y al describir la evaluación como un proceso complejo estamos separando 'cosas' que no se separan cuando se experimentan por un individuo.

Es decir, cuando conocemos evalúamos y lo hacemos a través de dos niveles básicos, que no existen el uno sin el otro. Es inevitable reconocer lo que estamos sintiendo, así como es inevitable construir sobre aquello que estamos sintiendo. Ahora bien, al hacerlo a través del lenguaje tenemos que tener en cuenta que la estructura del lenguaje, es radicalmente distinta a la estructura de aquello que intentamos conocer o explicar, el mundo de lo noverbal, el mundo de los 'hechos' (Caro, 1990b, 1994).
¿Por qué la estructura del lenguaje nos lleva fácilmente a conocer lo que nos sucede de una forma especial, cuyas consecuencias, a veces, no son todo lo deseables? La respuesta a esta cuestión se relaciona con el núcleo de la teoria y una de las metas básicas, en consecuencia, del tratamiento: la no identificación lenguaje-'realidad', o lo que es lo mismo, la distinción entre el mundo de lo verbal, de nuestras palabras, teorías, etc., y el mundo de lo no-verbal, el mundo de los "hechos', de nuestras experiencias, el mundo de lo no traducible a palabras. Digamos lo que digamos, pensemos lo que pensemos, NO ES nunca aquello que experimentamos (recordemos la primera premisa no aristotélica). Si el paciente no reconoce esta diferenciación decimos que está siguiendo un orientación intensional.

\section{La orientación intensional}

A lo largo de los años de trabajo con muestras clínicas, sobre todo pacientes que podríamos etiquetar con problemas de depresión y ansiedad, hemos comprobado que el uso del lenguaje incide en nuestra salud y que los pacientes tienen problemas cuando siguen lo que denominamos una orientación intensional, que refleja no reconocer las diferencias entre la estructura de los 'hechos' y del lenguaje.

Se manifiesta, ante todo, en lo que el paciente siente, se dice, y nos comunica. En nuestros estudios experimentales, sobre todo a través del análisis de tareas y a través de los comentarios de los pacientes hemos encontrado tres tendencias básicas que representan cómo aportamos significado a través del lenguaje (Caro, 1992, 1993a, 1996b).

A) Identificar, es decir, convertir palabras en 'hechos'. Se asume una actitud nominalista, confundiendo las palabras con los 'hechos'. Lingülsticamente se manifiesta, ante todo, y de forma más clara a través del verbo "ser", como nos aparece en etiquetas que un paciente depresivo se puede adscribir a sí mismo, como "soy un fracaso", etc.

B) Anticipar o seguir un orden equivocado de evaluación, es decir, anteponer palabras a 'hechos'. En lugar de centrarnos en el "aqui y ahora", saltamos a las conclusiones, trayen- 
do el futuro al presente y convirtiéndolo en una certeza (identificación). Es obvio recordar la inevitabilidad de hacer inferencias, de anticipar, de adelantarnos a los acontecimientos, pero muchos de nuestros problemas son consecuencia de tomar las anticipaciones como certezas y no como tales anticipaciones. Lingüisticamente, se manifiesta en enunciados con verbos en tiempo futuro, por ejemplo, "no tiene sentido que intente hacer esto, pues me va a salir mal".

C) Intensionalización que en cierta medida incluye a los otros dos, aunque lingüisticamente se manifiesta cuando utilizamos un lenguaje sin matices, absolutista, rígido. Hacemos intensionalizaciones cuando nos dejamos llevar por palabras, cuando nos movemos más en un nivel verbal de abstracción muy elevado. Por ejemplo, cuando decimos, "todo lo que he hecho lo he hecho mal" (el "todo" convierte a la frase en típicamente intensional).

La orientación intensional califica el tipo de evaluaciones que hacemos con más frecuencia. Al ser evaluaciones, no podemos olvidar que tienen un componente no-verbal y otro verbal. El aislar estos tres tipos de intensionalizaciones no significa que con la terapia lingüistica de evaluación los pacientes aprenden a clasificar aquello que se dicen a ellos mismos. Intentamos que se den cuenta de cómo aportan significado a los 'hechos', por regla general, de forma intensional, para 10 que, a veces, es necesario señalarles en las tareas que traen por escrito o bien al analizar situaciones por ellos vividas, qué significa hacer una identificación, o una anticipación, por ejemplo. Es decir, nos importa más que entiendan cómo se dejan llevar por las palabras, buscando como meta terapéutica, que los pacientes desarrollen una orientación extensional como veremos en breve.

\section{La depresión: un caso clínico}

Vamos a exponer de forma más extensa estas nociones sobre el lenguaje, empleando, para ello un caso clínico, que paso a detallar a continuación.

La paciente a la que llamaremos Silvia B. es diagnosticada por su psiquiatra como depresión mayor (episodio único grave), y nos es remitida por éste para ser tratada con la TLE. Las puntuaciones alcanzadas en las pruebas pretest y postest (1 de junio) aparecen en la tabla 1.

Tabla 1.- Puntuaciones de la paciente en las pruebas de pretest y postest

\begin{tabular}{|l|c|c|}
\hline Pruebas & Pretest & Postest \\
\hline HAD-A & 12 & 5 \\
HAD-D & 17 & 3 \\
BDI & 42 & 9 \\
BAI & 35 & 11 \\
STAI-E & 53 & 6 \\
STAI-R & 47 & 11 \\
PREO & 62 & 13 \\
& \\
\hline HAD: Escala Hospitalaria de Ansiedad y Depre- \\
sión; BDI: Inventario de Depresión de Beck y cols.: \\
BAl: Inventario de ansiedad de Beck y cols; STAl: \\
Inventario de Ansiedad Estado-Rasgo de \\
Spielberger y cols; PREO: Inventario de Preocu- \\
pación de Borkovec y cols. \\
\hline
\end{tabular}

\section{El proceso terapéutico}

Comienza el tratamiento el día 13 de Octubre de 1992, extendiéndose hasta el 1 de Junio de 1993, fecha en la que se le da el alta. El total de sesiones es de 24, una por semana (aunque no de una forma continuada), y de una hora de duración cada una de ellas.

Se organizan varias sesiones de seguimiento, en función de las posibilidades de paciente y terapeuta. La primera de ellas tiene lugar el día 29 de Junio (apreciándose una ligera recaida no significativa, y debida, según la paciente a su propio cansancio físico por su trabajo en casa). Ante esto se organiza una segunda sesión de seguimiento el 20 de Julio, en la que se aprecia una mejoria muy significativa, por encima, incluso de los niveles alcanzados en la fecha del alta.

Se hace una sesión de seguimiento con contacto telefónico el día 20 de octubre de 1993. La paciente confirma el mantenimiento de su mejoria y el estado emocional que alcanzó tras la terapia. 


\section{Descripción: sintomas y problemas principales}

Tal y como relata la paciente, en la Psicobiografía y en la primera sesión, sus problemas comenzaron, al casarse, y sobre todo después del último embarazo, dos años antes de acudir a consulta, por problemas en su casa materna, problemas con su marido, con su suegra, etc. En Noviembre del 91 (1 año antes del tratamiento psicológico) tuvo que recibir asistencia psiquiátrica por "encontrarme mal, fatigada, cansada, con desmayos y pérdidas de conocimiento". Estuvo ingresada un mes en el psiquiátrico por un intento de suicidio por ingestión de fármacos. Tuvo un segundo intento, no conocido por su familia, el verano de 1992 en el que, estando en la playa, se metió en el mar. Un bañista que nadaba cerca se acercó y la ayudó a salir.

Silvia a través de las entrevistas y las pruebas, manifiesta un antes y un despues. Antes del matrimonio y después. Antes, y aunque no tenia muy buenas relaciones con sus padres (sobre todo su padre), Silvia se describía como alegre, optimista, despreocupada, y con ganas de hacer cosas, la más alegre de su grupo, y la que proponía lo que se tenía que hacer por iniciativa propia. En el momento en que acude a consulta no le apetece estar con la gente, hacer cosas, sólo quiere estar sola, y dejar de hacer lo que los demás quieran. Desearia, "ser mejor madre, esposa, más optimista y más feliz", recuperar la alegría que perdió a raíz del matrimonio (lo describe como un desastre, con un marido que pasa de ella, que no le pregunta, le habla, ni es cariñoso, ni amable).

Esta situación se aprecia en una serie de sintomas depresivos de todo tipo, como carencia de actividades agradables, incapacidad para llevar a cabo las tareas de la casa (hijos, marido, etc.), triada cognitiva, pensamientos negativos ("pienso en morirme, desaparecer, en lo mal que lo hago todo, en las cosas que pueden ocurrir"), sentimientos negativos ("me siento, dolida conmigo misma, pesimista, como un trasto, insatisfecha"), autoculpabilidad, anhedonia, ausencia de relaciones sexuales, agresividad y discusiones con familiares, intentos de suicidio, etc.

\section{Conceptualización del caso}

Partiendo de la información recogida, se pudo conceptualizar el problema de la paciente, desde nuestra perspectiva teórica, de la siguiente manera.

Silvia se casó, según informa en las primera sesiones, "por salir de casa, llevar una vida mejor, más libre", reconociendo, en este momento, que no se cumplió lo que pensaba sobre el matrimonio cuando era joven. Es decir, desde la TLE podemos decir que Silvia hizo una evaluación fundamental, la identificación de matrimonio con libertad, una vida feliz y poder divertirse. A raíz de esta identificación vinieron una serie de anticipaciones que no se cumplieron (sigue atada a su familia y además atada a otra persona y a la familia de esa otra persona).

Se encontró, por tanto, en una situación en la que sus teorias previas, chocaban con sus 'hechos' presentes, los que reconstruía lingüisticamente de forma inapropiada, dejándose llevar por palabras y no reconociendo las circunstancias de los hechos, o las evaluaciones que hacía. Todo ello le lleva a estar metida es una espiral de intensionalizaciones (lenguaje absolutista, no condicional). A consecuencia de todas sus experiencias, la paciente ha desarrollado un concepto de ella misma muy negativo, realizando una serie de identificaciones que implicaban etiquetas negativas sobre ella misma, y cometiendo frecuentes "errores" de evaluación que fuimos analizando con la paciente en determinados momentos, algunos de los cuales ofrecemos a continuación, según la triple clasificación de la orientación intensional: "cuando salga de casa seré libre" (anticipación), "me caso y no soy libre" (reconocimiento de hechos), "no deberia haberme casado" (intensionalización), "estoy casada y no me comporto como tal" (identificación), "soy madre, pero no ejerzo" (identificación), "debería encontrarme a gusto en casa" (intensionalización), "debería comportarme como madre" (intensionalización), etc.

\section{CAMBIO}

El procedimiento terapéutico de la terapia lingüistica de evaluación se plantea las siguientes metas. En primer lugar, el cambio terapéutico postulado y buscado es un cambio de tipo 
lingüistico, es decir, el paciente cambia en su forma de hablar sobre las cosas, to que le sucede o experimenta. De forma más concreta, lo que se busca es conseguir un cambio desde lo intensional hasta la extensional, cambio éste que supone un ajuste en estructura entre el mundo de los 'hechos' y el mundo del lenguaje. Es decir, el lenguaje es elementalista, separa cosas que no se pueden separar (por ejemplo, 'cognición' de 'emoción'), y tiene un menor grado de diferenciación, riqueza y variabilidad que el mundo de lo no-verbal, de los 'hechos'. En definitiva con la terapia buscamos que el paciente desarrolle una orientación extensional, la opuesta a la intensional ya expuesta y que analizaremos a continuación.

\section{La orientación extensional}

Tenemos una actitud extensional cuando no identificamos, no anticipamos y hacemos extensionalizaciones, es decir, entramos en contacto con los 'hechos' de forma continua. Intentamos, pues, que la persona desarrolle una actitud distinta frente al uso del lenguaje, reconociendo, sobre todo, lo que suele ser una saludable diferenciación: aquella que se establece entre palabras y 'hechos'. La búsqueda de esta diferenciación se fundamenta en que la estructura del lenguaje es diferente de la estructura de lo no- verbal.

La extensionalización propiamente dicha es estar en contacto con los 'hechos', sin poner palabras, pero eso es imposible, por eso consideramos que cuando los pacientes hacen extensionalizaciones nos ofrecen siempre elaboraciones, matizaciones, precisiones sobre las cosas. Un ejemplo de lenguaje no absolutista, condicional, flexible (extensionalización) seria, "he pensado que debo recapacitar sobre mi vida y actuar a partir de ahora contando con lo que tengo hoy, y no pensando en lo que yo queria hace 10 años". Un ejemplo de discriminación entre palabras y 'hechos' (no identificación) seria, "no soy una mala madre, pues en mayor o menor medida tengo bien cuidados a mis hijos. $Y$ si no puedo es a causa de mi depresión". Finalmente un ejemplo en el que se toma a las anticipaciones como tales y no como certezas (no anticipación) seria, "me encuentro mal por que va a llegar mi marido. No deberia anticipar si no sé lo que va a pasar luego".

Hemos visto las puntuaciones pretest y postest. ¿Ahora bien cómo cambió la paciente? Para demostrar que el cambio lingüístico se ha producido desde lo intensional hacia lo extensional, vamos a analizar el tipo de intensionalizaciones y extensionalizaciones realizadas por Silvia en dos acontecimientos seleccionados, de la sesión $7^{a}$, que representan, quizá, una identificación importante, el núcleo de buena parte de sus problemas. Seguimos para ello, la metodologia del análisis de tareas que ya hemos utilizado para analizar acontecimientos $y$ ofrecer un primer modelo del cambio en una paciente con problemas etiquetados de ansiedad (Caro, 1996b).

\section{Método \\ El análisis del proceso del cambio: el análisis de tareas}

En años recientes ha cobrado una gran importancia entender el cambio terapéutico desde una perspectiva procesual. En lugar de evaluar qué cambios se producen con la psicoterapia, lo que se pretende es entender cómo cambian los pacientes a lo largo del curso de la terapia. Son muy diversas las metodologias que nos permiten describir, explicar y predecir el cambio terapéutico (cf. Caro, 1993b). En este contexto hemos elegido la metodologia del análisis de tareas que ha contribuido y se ha beneficiado a su vez de la tendencia actual hacia el estudio de los acontecimientos terapéuticos.

Esta perspectiva presta atención al momento exacto, intervalo temporal y el lugar o secuencia en una o más sesiones terapéuticas en los que se espera que comiencen procesos de cambio importantes del paciente o aquellos que ya han comenzado, de forma tal que se puedan analizar las modalidades o condiciones de estos cambios (Fiedler, 1995).

El análisis de tareas (Greenberg, 1984; 1991; Safran, Greenberg y Rice, 1988) combina una metodología racional y empirica que pretende descubrir cuáles son los componentes de la competencia implicada en las ejecuciones de determinada tarea para poder entender cómo se resuelve un problema. En otros términos podemos considerar que supone la 
investigación intensiva de caso único, con replicación. En el análisis de tareas no se estudian individuos, sino ejecuciones resueltas siguiendo la misma estrategia, con la idea de encontrar el modelo de las operaciones cognitivas que conducen al cambio buscando las operaciones del paciente, del terapeuta o de ambos.

Las fases en el análisis de tareas son ocho (Greenberg, 1991). En la primera fase se hace un mapa, es decir, se articulan los hechos que se deben dar en terapia para resolver un problema, y en la segunda fase se especifica la tarea/ambiente de la tarea (conceptos que se van a medir en un ambiente estable). Ambas corresponderian a la conceptualización de la evaluación como aquil se ha expuesto y a la posibilidad de cambio mediante la técnica de los órdenes de abstracción. En la tercera se verifica la significación de la tarea, es decir, se empiezan a encontrar los ingredientes del cambio, algo que ya hemos comenzado a hacer en otros trabajos (Caro, 1992, 1993a, 1996b), para lo que se necesita haber demostrado la eficacia terapéutica del procedimiento (Caro, 1992). En la fase cuatro se hace un primer análisis racional (diagramas de ejecución) o un modelo de resolución de la tarea en concreto. Con este trabajo presentamos datos que continuan nuestros trabajos anteriores y que suponen replicar lo encontrado sobre el concepto de evaluación y su relación con la orientación intensional vs. extensional, correspondiendo los datos aquí presentados a las fases 5 y 6 del análisis de tareas. En la fase 5 se hace un análisis empirico (descripción de ejecuciones reales tal y como luego se expondrá), y en la fase 6 se comparan ejecuciones reales y posibles para construir un modelo específico (modelo de resolución que se expone en la figura 1). Las dos últimas fases corresponden a la validación (fase 7 donde se comprueban hipótesis sobre ejecuciones de clientes utilizando datos grupales y comparando ejecuciones resueltas y no resueltas), en nuestro caso seria posible hacerla ampliando el número de acontecimiento resueltos y no resueltos. En la fase última, la 8 se relaciona proceso con resultado, es decir, se demuestra estadísticamente que los resultados alcanzados vienen dados por la ejecución de determinadas tareas terapéuticas. En nuestro caso sólo con la comparación grupal es posible correlacionar elementos que inciden en el cambio con el cambio final.

Dada la muestra analizada hemos llegado hasta aqui a la fase 6 , y no a la 7 y 8 que sólo son posibles con un análisis grupal y que ya hemos realizado en otro trabajo (Caro, 1996b).

\section{Selección de acontecimientos}

Los acontecimientos analizados pueden provenir de diversos pacientes, o del mismo, analizado de forma intensiva. Los acontecimientos, suelen ser buenos ejemplos en los que se aprecie cómo el paciente (asistido por el terapeuta) resuelve una tarea terapéutica. Lógicamente, es deseable disponer de otros acontecimientos, a su vez, buenos ejemplos de como el paciente NO resuelve una tarea terapéutica.

Una tarea es una serie completa de intervención psicológica, y debe cumplir una serie de requisitos

1. Marcador definido teóricamente (problemas del paciente, sintomas, crisis, etc.): En nuestro caso "Rechazo a la casa".

2. Una o más operaciones terapéuticas conceptualmente esperadas (estrategias, técnicas, métodos): La técnica de los "Ordenes de abstracción". Una de las técnicas básicas de la TLE.

3. La conducta de ejecución de un paciente que sigue a una intervención: Acontecimientos no resueltos y resueltos.

4. La estimación de los efectos directos de los intentos de solución del problema (resultado en sesión): Cambio de lo intensional a lo extensional.

Para llevar a cabo el análisis, y seleccionar tareas y acontecimientos, se escuchan sesiones en las que estos acontecimientos aparecen, se seleccionan en función de los criterios que ahora se señalarán se procede a su transcripción, y después, se pasan por el microscopio del análisis de tareas.

Los dos acontecimientos aquí analizados intensivamente, se seleccionaron, como ya he dicho por representar muy bien, la evaluación que estaba detrás de buena parte de los problemas de la paciente. La situación era la siguiente: la paciente entraba en casa y la 
evaluaba negativamente, iba camino de casa y se ponia a llorar, o entraba en casa y se encontraba mal, sin ganas de hacer nada, discriminando la diferencia de sentimientos que tenía cuando estaba en su casa, sobre todo con su marido, y fuera de ella, sobre todo sola (se encontraba bien, incluso muy bien). Acordamos, en sesión, hablar de ese tema y lo hacemos utilizando la técnica (ambiente de la tarea) de los órdenes de abstracción, una de las técnicas semántico-generales que empleamos con nuestros pacientes (Caro, 1994).

Un ejemplo de la técnica nos aparece en el cuadro 1.

\section{No resolución vs. resolución}

Los órdenes representan un proceso inductivo que va desde lo no-verbal hasta lo verbal. Esto es, le pedimos a una persona, primero para centrarla, que diga que le pasa por la cabeza en determinada situación, aquella que quiere resolver, después, que diga cómo se siente en una situación, que la valore y luego pase a analizarla nivel a nivel: etiqueta, descripción, inferencias, conclusión, y que la valore, de nuevo, a la luz de la nueva conclusión. Tal y como está preparada nos sirve para dar marco al debate encaminado a que el paciente ajuste la estructura de lo que dice

Cuadro 1. Ejemplo de la tarea de los órdenes de abstracción

NOMBRE:

FECHA:

La tarea que va a hacer a continuación le permitirá resolver situaciones problemáticas que le ocurren con frecuencia. Pero para ello debe hacerla en el momento en que tiene alguna idea negativa.

Ante determinada situación/hecho a usted le asaltan ideas repetitivas, negativas, que le hacen encontrarse mal. Le pedimos, que anote LA SITUACION/EL HECHO ante el que le aparecen estas ideas:

IDEAS NEGATIVAS:

ANOTE COMO LE HACEN ENCONTRARSE:

Muy mal: $\quad-5-4-3-2-10+1+2+3+4+5 \quad$ Muy bien

A continuación, le pedimos que:

Anote lo que ESTA EXPERIMENTANDO EN EL MOMENTO PRESENTE, LO QUE ESTA VIVIENDO AQUI Y AHORA (la situación concreta, el hecho concreto):

Ponga una ETIQUETA (la palabra o palabras que en su opinión mejor resumiría o describiría lo que está experimentando, la situación en la que está):

¿Puede DESCRIBIR la situación?:

A partir de ello, por favor, y en relación a la situación concreta en la que está, piense sobre ello: ¿QUE DEDUCE?, ¿QUE LE PASA POR LA CABEZA CON MAS FRECUENCIA?, ¿QUE PIENSA DE TODO ELLO?, ¿COMO LO PUEDE RESOLVER?, ¿COMO LO PUEDE VER DE OTRA MANERA?:

Finalmente, ¿QUE CONCLUSION PUEDE SACAR DE TODO ELLO?:

ANOTE COMO LE HACE ENCONTRARSE EL HABER SEGUIDO ESE PROCESO:

Muy mal: $\quad-5-4-3-2-10+1+2+3+4+5 \quad$ Muy bien 
a la estructura de lo que experimenta. Además, cuando la aprenden los pacientes, la utilizan (en el formato que se presenta en el cuadro 1) como tarea extrasesión.

Expliquemos brevemente lo que pasó en este caso. En el orden no resuelto comenzamos con las ideas negativas siguientes asociadas con la sensación de estar a disgusto en casa: "debería sentirme igual en casa, debería sentirme mejor, pero llego a casa y me siento peor". En este sentido la paciente valoraba la situación como -5 (ver cuadro 1). Esto le lleva a etiquetarse como "idiota" y decirse que "debo pasar de todo". Y acaba concluyendo "debo pasar de todo, no hacer caso de nada". Valora el resultado final como -3 .

Dado que la paciente saca conclusiones en forma de intensionalizaciones, acordamos con ella seguir analizando esa situación con la tarea de los órdenes y esta vez la paciente termina resolviendo la situación, concluyendo: "debería tratar de ver a mi marido y no a mi suegra cuando miro a mi marido". Valorando esta vez la paciente todo con +3 .

Sabemos el antes y el después, pero cómo - por qué procesos, qué operaciones están implicadas en la no resolución o resolución, o cómo alcanzó la $P$. esas conclusiones.

Partimos de dos hipótesis básicas:

\section{Hipótesis básicas}

1. Durante la no resolución habrá un mayor número de intensionalizaciones y de extensionalizaciones durante la resolución. Es decir, la resolución estará asociada, en la paciente, con un ajuste estructural lenguaje-'hechos', mientras que la no resolución lo estará con un desajuste.

2. La ejecución resuelta y la no resuelta estará asociada con un tipo determinado de operaciones terapéuticas, tanto del paciente como del terapeuta.

\section{Procedimiento de análisis de los acontecimientos}

Para encontrar las operaciones que diferencian la resolución y la no resolución y la extensionalización y la intensionalización, clasificamos las intervenciones de la paciente y de la terapeuta en los dos acontecimientos, analizados con los órdenes de abstracción, en enunciados con sentido. La $\mathrm{N}$ total fue de:
336, desglosada de la siguiente forma: Paciente: No resuelto $=50$ enunciados; terapeuta: No resuelto $=58$ enunciados; paciente: Resuelto $=116$ enunciados; terapeuta: Resuelto $=112$ enunciados.

Se buscan trabajando con las transcripciones, las operaciones de la paciente y de la terapeuta. Es decir, lo que representa aquello que hace la paciente y aquello que hace la terapeuta. Todo lo que se dice en un acontecimiento es importante y por eso se categorizan todos y cada uno de los enunciados. La idea es "descubrir" dichas operaciones, poniendo nombre (con la ayuda del marco terapéutico) a todo lo que se hace en la tarea analizada. Como veremos a continuación hemos encontrado operaciones relacionadas con problemas de evaluación y otras consecuencia de la propia estructura de la terapia, o de la técnica de los órdenes de abstracción. Por regla general, y para evitar errores se categorizan, primero, usando categorlas muy descriptivas, por ejemplo, "rechazo a la suegra". En una segunda lectura se puede pasar a perfilar más (abstraer) estas categorias "Evaluación negativa". Este trabajo corresponde a la fase quinta de la metodología del análisis de tareas, y en base a él podemos comparar la resolución y la no resolución y hacer el diagrama de resolución (fase 6) en base a las principales operaciones encontradas.

Para controlar posibles sesgos se buscó un acuerdo entre jueces (nota 1), aunque sólo en relación a la categorización de las operaciones de la paciente. El acuerdo, medido a través de la Kappa de Cohen, fue de .8106, para las categorias del acontecimiento no resuelto y de 8496 para las categorias del acontecimiento resuelto. Dichas cifras son altamente satisfactorias, por lo que consideramos el procedimiento de categorización terminado. Hasta el momento de la búsqueda del acuerdo entre jueces, las operaciones de paciente y terapeuta se habian revisado 7 veces. A pesar de eso, hubo una última revisión en la que se cambiaron, unos pocos códigos, en función de los códigos de la otra juez.

\section{Análisis}

Dado el tipo de datos, hicimos un recuento de frecuencias en cada categoría u operación. y posteriormente, porcentajes. 
Resultados: Principales operaciones encontradas

Operaciones de la paciente

1. Intensionalización: intensionalizaciones propiamente dichas (es decir, el uso de un lenguaje absolutista) e identificaciones (la paciente no hace anticipaciones que es más propio de pacientes con problemas de ansiedad).

Aparecen aqui todos los enunciados de la paciente como "debería encontrarme bien en casa", "debería estar siempre bien", "soy idiota", "debería pasar de todo"; "mi marido es culpable", etc.

2. No identificación: cuando la paciente se da cuenta que confunde a su marido con su suegra y que eso motivaba su rechazo a su casa.

3. Evaluación negativa: rechazo hacia la suegra, y la casa; Valoración entre -1 y -5 en los órdenes; diferencia entre situaciones (cuando la paciente comenta fuera de casa me encuentro bien y dentro mal).

4. Evaluación positiva: aceptación de personas o situaciones; Valoración entre +1 y +5 en los órdenes.

5. Reconoce teorias: cuando la paciente se da cuenta que aporta palabras a las cosas.

6. Condicional: cuando la paciente se da cuenta qué explica, o de qué dependen sus reacciones.

7. Descripción/etiqueta: representan un uso extensional del lenguaje, a través de dar "descripciones", "etiquetas ajustadas" y "definir acciones".

8. Ventaja de la extensionalización: Efectos positivos y anticipaciones positivas después de sacar conclusiones más extensionales. Por ejemplo, "si logro dejar a la suegra fuera, va a mejorar mi relación con mi familia".

9. Dudas: todas las respuestas de la paciente que empiezan con "no sé", "no sé qué decirte, cómo definirlo", etc.

10. Perplejidad: cuando la paciente manifiesta que no se entiende a ella misma, ni sus reacciones, etc.

11. Acuerdo: la paciente manifiesta su acuerdo a sugerencias o comentarios de la terapeuta.

12. Comentarios marginales: se comentan temas (junto a la terapeuta) que luego no entran en el debate con los órdenes. Por ejemplo, la paciente comenta que cualquier dia la vecina de arriba la va a denunciar por que sus hijos hacen ruido"
Operaciones de la terapeuta

1. Explorar: búsqueda de pensamientos, a raiz de esta operación es cuando salen la mayoria de las intensionalizaciones de la paciente.

2. No identificación: trabaja la terapeuta intentando que la paciente vea cuál era su identificación básica, en este caso la identificación entre su suegra y el marido. A esta operación le seguia los "no se" de la paciente, hasta que logra las "no identificaciones".

3. Búsqueda de la conclusión: corresponde a la parte final de los órdenes, y representa pedirle a la paciente una o varias conclusiones para dar por cerrado el debate. Va seguida de "intensionalizaciones" e "identificaciones" en la no resolución y de "no identificaciones" en la resolución.

4. Valoración: la terapeuta le pide que valore con los órdenes lo que se está trabajando, o que comente su apreciación o no de su suegra. Va seguida, por parte de la paciente de "evaluaciones negativas" o "positivas".

5. Señala teorias: la terapeuta le hace ver a la paciente que está aportando palabras a 'hechos'. En este caso la paciente las "reconoce", o "está de acuerdo".

6. Condicional: se le pide a la paciente que vea los motivos, lo que explica o de qué dependen sus reacciones. La paciente puede ofrecer un "condicional", o manifestar su "perplejidad".

7. Trabajo con la etiqueta/descripción: la terapeuta intenta anular las etiquetas negativas de la paciente, bien haciendo que haga descripciones o que defina acciones.

8. Ventajas de la extensionalización: se le pide a la paciente que diga cómo se encuentra o qué puede anticipar, positivamente hablando, después de haber sacado una conclusión de tipo más extensional.

9. Reflejo: entendido en un sentido estricto psicológico, es decir, poner palabras a la experiencia de la paciente. Puede ir seguido por "acuerdo" de la paciente.

10. Aspectos técnicos: concretar o centrar a la paciente en la tarea; explicar algunos elementos de la terapia, por ejemplo recordarle a la paciente que es una etiqueta; repaso, siguiendo la estructura de los órdenes de lo tratado hasta el momento. Suele ir seguida de "acuerdo. 
11. Apoyo: La terapeuta manifiesta su "acuerdo" con lo que dice la paciente, y hace "refuerzo verbal", el tradicional "Mhm" de los terapeutas.

12. Comentarios marginales: Paralelos a los de la paciente. Comentarios no relevantes para el debate. Como por ejemplo, "por qué otro motivo te va a denunciar la vecina de arriba".

\section{Principales resultados}

Operaciones de la paciente

1) Respecto a nuestra primera hipótesis, que la intensionalización $(62.5 \%)$ (con "intensionalizaciones" propiamente dichas e "identificaciones") caracteriza a la no resolución y la extensionalización (100\%)(con "no identificaciones" exclusivamente) a la resolución, vemos que se cumple, aunque aparecen inten-

Tabla 2.- Principales operaciones de la paciente y de la terapeuta

\begin{tabular}{|c|c|c|c|c|c|}
\hline \multirow{2}{*}{$\begin{array}{l}\text { OPERACIONES } \\
\text { PACIENTE }\end{array}$} & \multirow{2}{*}{$\frac{\Sigma \text { y \% }}{\text { No Resuelto }}$} & \multirow{2}{*}{$\frac{\sum \text { y \% }}{\text { Resuelto }}$} & \multirow{2}{*}{$\begin{array}{l}\text { OPERACIONES } \\
\text { TERAPEUTA }\end{array}$} & $\Sigma$ y \% & $\Sigma$ y $\%$ \\
\hline & & & & No Resuelto & Resuelto \\
\hline INTENSIONALIZACION & $\begin{array}{l}20 \\
62.5 \%\end{array}$ & $\begin{array}{l}12 \\
37.5 \%\end{array}$ & EXPLORAR & $\begin{array}{l}15 \\
60 \%\end{array}$ & $\begin{array}{l}10 \\
40 \%\end{array}$ \\
\hline NO IDENTIFICACION & - & $\begin{array}{l}14 \\
100 \%\end{array}$ & $\begin{array}{l}\text { NO IDENTI- } \\
\text { FICACIONN }\end{array}$ & - & $\begin{array}{l}11 \\
100 \%\end{array}$ \\
\hline EVALUACION NEGATIVA & $\begin{array}{l}12 \\
46.15 \%\end{array}$ & $\begin{array}{l}14 \\
53.85 \%\end{array}$ & $\begin{array}{l}\text { BÚSQUEDA } \\
\text { CONCLUSIÓN }\end{array}$ & $\begin{array}{l}3 \\
42.86 \%\end{array}$ & $\begin{array}{l}4 \\
57.14 \%\end{array}$ \\
\hline EVALUACION POSITIVA & $\begin{array}{l}1 \\
14.29 \%\end{array}$ & $\begin{array}{l}6 \\
85.71 \%\end{array}$ & VALORACIÓN & $\begin{array}{l}6 \\
40 \%\end{array}$ & $\begin{array}{l}9 \\
60 \%\end{array}$ \\
\hline RECONOCE USO LENGUAJE & $\begin{array}{l}3 \\
42.86 \%\end{array}$ & $\begin{array}{l}4 \\
57.14 \%\end{array}$ & $\begin{array}{l}\text { SEÑALA USO } \\
\text { LENGUAJE }\end{array}$ & $\begin{array}{l}6 \\
40 \%\end{array}$ & $\begin{array}{l}9 \\
60 \%\end{array}$ \\
\hline CONDICIONAL & 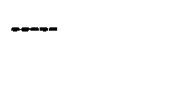 & $\begin{array}{l}6 \\
100 \%\end{array}$ & CONDICIONAL & $\begin{array}{l}3 \\
50 \%\end{array}$ & $\begin{array}{l}3 \\
50 \%\end{array}$ \\
\hline ETIQUETAS/DESCRIPCION & $\begin{array}{l}1 \\
5 \%\end{array}$ & $\begin{array}{l}19 \\
95 \%\end{array}$ & $\begin{array}{l}\text { TRABAJO } \\
\text { ETIQ./ DESCR. }\end{array}$ & $\begin{array}{l}2 \\
7.41 \%\end{array}$ & $\begin{array}{l}25 \\
92.59 \%\end{array}$ \\
\hline VENTAJAS EXTENSIONAL & ---- & $\begin{array}{l}7 \\
100 \%\end{array}$ & $\begin{array}{l}\text { VENTAJAS } \\
\text { EXTENSIONAL }\end{array}$ & - & $\begin{array}{l}3 \\
100 \%\end{array}$ \\
\hline DUDAS & $\begin{array}{l}7 \\
43.75 \%\end{array}$ & $\begin{array}{l}9 \\
56.25 \%\end{array}$ & REFLEJO & $\begin{array}{l}5 \\
38.46 \%\end{array}$ & $\begin{array}{l}8 \\
61.54 \%\end{array}$ \\
\hline PERPLEJIDAD & $\ldots$ & $\begin{array}{l}6 \\
100 \%\end{array}$ & $\begin{array}{l}\text { ASPECTOS } \\
\text { TÉCNICOS }\end{array}$ & $\begin{array}{l}7 \\
63.64 \%\end{array}$ & $\begin{array}{l}4 \\
36.36 \%\end{array}$ \\
\hline ACUERDO & $\begin{array}{l}4 \\
26.67 \%\end{array}$ & $\begin{array}{l}11 \\
73.33 \%\end{array}$ & APOYO & $\begin{array}{l}9 \\
32.14 \%\end{array}$ & $\begin{array}{l}19 \\
67.86 \%\end{array}$ \\
\hline COMENTARIO MARGINAL & $\begin{array}{l}2 \\
20 \%\end{array}$ & $\begin{array}{l}8 \\
80 \%\end{array}$ & $\begin{array}{l}\text { COMENTARIO } \\
\text { MARGINAL }\end{array}$ & $\begin{array}{l}2 \\
22.22 \%\end{array}$ & $\begin{array}{l}7 \\
77.78 \%\end{array}$ \\
\hline
\end{tabular}


PACIENTE

\begin{tabular}{|c|}
\hline Acuerdo \\
\hline $\begin{array}{l}\text { Intensionalizaciones } \\
\text { "debería estar a gusto" } \\
\text { "deberia sentirme mejor" }\end{array}$ \\
\hline Valoración: -5 \\
\hline $\begin{array}{l}\text { Etiqueta: "soy idiota", } \\
\text { "tendria que pasar de } \\
\text { todo" }\end{array}$ \\
\hline $\begin{array}{l}\text { Intensionalizaciones } \\
\text { "Deberia..." }\end{array}$ \\
\hline $\begin{array}{l}\text { Conclusión negativa: } \\
\text { "Debo pasar de todo" } \\
\text { Valoración: }-3\end{array}$ \\
\hline $\begin{array}{l}\text { Descripción } \\
\text { Dudas } \\
\text { Perplejidad }\end{array}$ \\
\hline $\begin{array}{l}\text { "No soporto estar con } \\
\text { mi marido" }\end{array}$ \\
\hline $\begin{array}{l}\text { Culpabilidad del marido: } \\
\text { Dudas } \\
\text { Perplejidad }\end{array}$ \\
\hline $\begin{array}{l}\text { Descripciones } \\
\text { Condiciones }\end{array}$ \\
\hline $\begin{array}{l}\text { Reconocimiento de la } \\
\text { Identificación }\end{array}$ \\
\hline $\begin{array}{l}\text { Evaluación +: marido } \\
\text { Evaluación -: suegra }\end{array}$ \\
\hline $\begin{array}{l}\text { Conclusión extensional: } \\
\text { "dejar a mi suegra fuera } \\
\text { de casa" } \\
\text { Valoración: }+3\end{array}$ \\
\hline
\end{tabular}

TERAPEUTA

\section{NO RESOLUCIÓN}

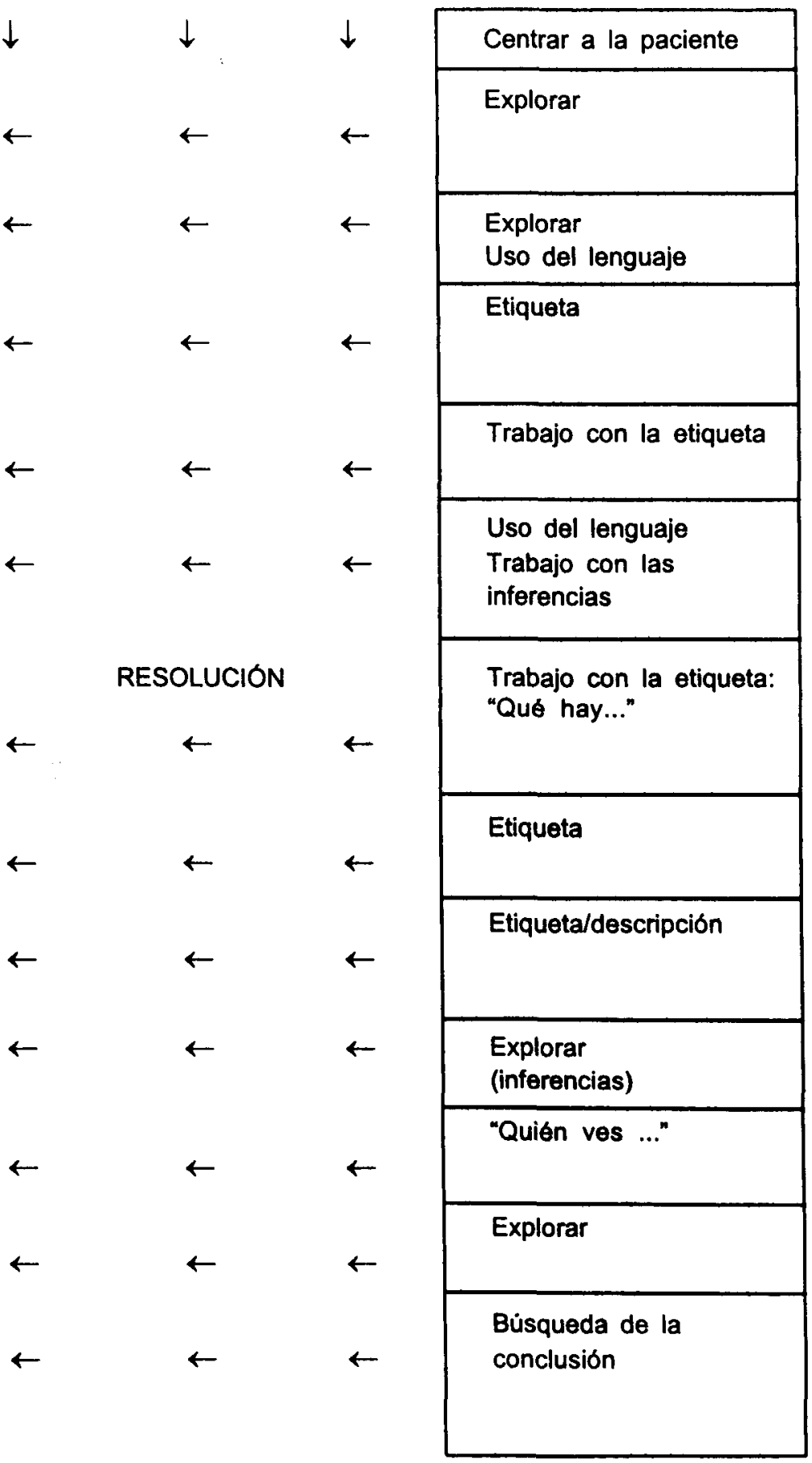

Nota. La figura se lee de arriba a abajo y de derecha a izquierda. Por ejemplo, la terapeuta centra en el tema a tratar a la paciente y ésta está de acuerdo, luego la terapeuta pasa a explorar y ahí surgen intensionalizaciones, etc. 
sionalizaciones en la resolución $(37,5 \%)$ no hay ningún tipo de extensionalización en la no resolución (ver tabla 2 ).

2) ¿Qué tipo de operaciones están implicadas en la no resolución y en la resolución? Al margen de que durante la no resolución hay un mayor número de "intensionalizaciones", e "identificaciones" y en la resolución lo hay de "no identificaciones", como ya hemos visto, aparecen una serie de operaciones clave.

En la no resolución $(46,15 \%)$ y en la resolución $(53,85 \%)$ hay un número semejante de "Evaluaciones negativas", que corresponden al "rechazo de la situación en la que se encuentra" y el "rechazo hacia su suegra". Mientras que hay una "evaluación positiva" $(14,29 \%)$ en la no resolución, y estas aumentan en la resolución $(85,71 \%)$, manifestadas a través de la "aceptación del marido" y de "valoraciones positivas", propias de la resolución.

En ambos acontecimientos $(42,86 \%$ y $57,14 \%$, respectivamente) a instancias de la terapeuta, la paciente reconoce el "uso del lenguaje", es decir, que aporta teorias a las cosas.

Sin embargo, las siguientes operaciones son más relevantes para la resolución. El "condicional" sólo aparece en el acontecimiento resuelto $(100 \%)$, mientras que la paciente se mueve a niveles descriptivos ("trabajo con la etiqueta/descripción") en un número bastante más elevado en la resolución (95\%). Lógicamente, también, es durante el acontecimiento resuelto en donde la paciente puede percibir los "efectos beneficiosos" de la extensionalización $(100 \%)$, desde "encontrarse mejor al sacar una conclusión más extensional, a hacer "anticipaciones positivas" sobre las ventajas de la no identificación.

Las dos siguientes categorias, manifiestan lo difícil que es esa situación para la paciente. Así, es común que la paciente no sepa que contestar ("dudas") en ambos acontecimientos $(43,75 \%$ y $56,25 \%)$ a preguntas que le hacía la terapeuta. Al explorar más, en el acontecimiento resuelto las situaciones y sus reacciones, es lógico que la paciente manifieste en este una sensación de "perplejidad" (100\%).

Finalmente, hay "acuerdo" en ambos acontecimientos $(26,67 \%)$, aunque más $(73,33 \%)$ en la resolución. $E$ igualmente, un mayor número de "comentarios marginales" (20\% y $80 \%$ respectivamente).
Operaciones de la terapeuta

1) Salvo dos, la mayoria de las operaciones de la terapeuta aparecen en los dos acontecimientos. Aunque hay más Exploración en la no resolución (60\%), la "búsqueda de la no identificación" sólo es evidente en la resolución $(100 \%)$. Mientras que la búsqueda de conclusiones es prácticamente igual en ambos acontecimientos $(42 ; 86 \%$ y $57,14 \%)$.

La "valoración" (pedirle a la paciente la valoración antes y después del orden, y pedirle su opinión sobre la suegra) es un poco más elevada durante la resolución ( $40 \%$ y $60 \%$ ) y también la terapeuta le" señala el uso del lenguaje en un porcentaje mayor en el acontecimiento de resolución ( $40 \%$ y $60 \%$ ).

Respecto al "condicional" los porcentaje son exactamente iguales (50\% y $50 \%$ ). Sin embargo, trabaja más con las etiquetas y las descripciones en la resolución $(7,41 \%$ y $92,59 \%)$.

Lógicamente, exclusiva de la resolución és señalarle a la paciente la "ventajas de la extensionalización" (100\%).

Finalmente, el "reflejo" es mayor en el acontecimiento resuelto $(38,46 \%$ y $61,54 \%)$, los "aspectos técnicos" son un poco más elevados en la no resolución $(63,64 \%$ y $36,36 \%)$. El "apoyo" es mayor durante la resolución $(32,14 \%$ y $67,86 \%)$. $Y$, finalmente, los "comentarios marginales" son mayores durante la resolución $(22,22 \%$ y $77,78 \%)$.

Cómo se relacionan, procesualmente hablando, las operaciones anteriores. Un elemento básico del procedimiento del análisis de tareas ( $6^{\text {a }}$ fase) es hacer un diagrama que dibuje o describa el cambio que tuvo lugar (ver figura 1).

\section{Discusión}

Varias cuestiones cabe señalar referidas a los resultados obtenidos con la paciente.

En primer lugar, desde luego, la intensionalización es más propia de la no resolución aunque no es ajena a los acontecimientos donde se resuelve una tarea. Resultado que concuerda con otros de anteriores estudios (Caro, 1993, 1996b). Hay que tener en cuenta, que paciente y terapeuta hablan y elaboran el tema sobre el que se esté discutiendo y que eso supone ya de por si la aparición de evalua- 
ciones concretas, manifestadas con un lenguaje de tipo intensional, $e$ incluso de una cierta sensación de perplejidad, en este caso.

En segundo lugar, respecto a las operaciones típicas de la resolución tenemos las no identificaciones, relacionadas todas ellas con la anulación de la identificación básica.

Llama la atención el aumento en el número de frecuencias referidas a las descripciones y etiquetas, que es comprensible si vemos las operaciones de la terapeuta y la importancia que tiene trabajar con descripciones de situaciones concretas, diferencias entre personas, etc.

El logro de la no identificación sólo es posible cuando paciente y terapeuta han descrito lo que ocurre y han planteado el tipo de inferencias que hace la paciente en determinadas situaciones, más o menos recientes, que ocurrieron en su casa, con la idea de entender qué tipo de palabras no ajustadas estructuralmente está aportando la paciente.
Nos aparece claramente a través de la resolución de una situación problemática las ventajas de la extensionalización. Korzybski (1941) asumió que todas las psicoterapias enseñaban a los pacientes a extensionalizar y que esto suponia hacerlos entrar en contacto con los 'hechos', discriminando entre estos y las palabras. Qué discriminó y qué extensionalizó la paciente.

Releyendo sobre los datos que acabamos de presentar podemos postular que la paciente llevó a cabo un ajuste estructural 'hechos'palabras, en la línea reflejada en el cuadro 2.

En definitiva, la paciente la situación no se resolvía por qué no se había encontrado la evaluación básica, en forma de identificación que explicaba su rechazo a la casa.Qué es necesario para encontrarla: básicamente explorar bien lo que piensa un paciente ante una situación y tener claro el tipo de palabras que aporta al respecto las evaluaciones implicadas y cómo le hacen estas sentirse.

Cuadro 2.- Explicación del cambio terapéutico

\begin{tabular}{|c|c|}
\hline \multicolumn{2}{|c|}{ NO AJUSTE ESTRUCTURAL } \\
\hline SITUACIONES & PALABRAS INTENSIONALES \\
\hline $\begin{array}{l}\text { ODIO HACIA LA SUEGRA } \\
\text { RECHAZO A LA CASA } \\
\text { RECHAZO AL MARIDO } \\
\text { BLOQUEO EN UNA SITUACION } \\
\text { NO SABER QUE HACER } \\
\text { ESTAR PREOCUPADA }\end{array}$ & $\begin{array}{l}\text { "Soy culpable" } \\
\text { "Debería sentirme bien" } \\
\text { "Deberia comportarse de otro modo" } \\
\text { "Debería pasar de todo" } \\
\text { "No paso de todo" } \\
\text { "Soy idiota" }\end{array}$ \\
\hline \multicolumn{2}{|c|}{ AJUSTE ESTRUCTURAL } \\
\hline SITUACIONES & PALABRAS MAS EXTENSIONALES \\
\hline ODIO HACIA LA SUEGRA & $\begin{array}{l}\text { "No soy culpable. No la quiero y no } \\
\text { me quiere. Me alegro de haberme com- } \\
\text { portado asi" }\end{array}$ \\
\hline QUIEN ESTA EN CASA & $\begin{array}{l}\text { "Marido, hijos" } \\
\text { "Mi marido NO ES su madre" }\end{array}$ \\
\hline $\begin{array}{l}\text { "PERSPECTIVA" } \\
\text { QUE HACER }\end{array}$ & $\begin{array}{l}\text { "Dejar fuera de casa a mi suegra" } \\
\text { "Mejorará la relación con mi marido y } \\
\text { toda la situación en casa" }\end{array}$ \\
\hline
\end{tabular}

